\title{
On the Existence of Crossing Symmetric and Unitary Scattering Amplitudes with Regge Poles
}

\author{
J. Kupsch \\ Fachbereich Physik, Universität Kaiserslautern, D-6750 Kaiserslautern, \\ Federal Republic of Germany
}

\begin{abstract}
We give a complete proof of the existence of scattering amplitudes $A(s, t, u)$ with the following properties

i) the amplitudes are total symmetric in $s, t$, and $u$,

ii) they satisfy elastic unitarity for $4 \leqq s \leqq 16$, and

iii) they develop resonances for $l \geqq 2$ on a bounded Regge trajectory which dominates the asymptotics for large energies.
\end{abstract}

\section{Introduction}

A rather general class of $\pi \pi$ scattering amplitudes which satisfy exactly crossing symmetry elastic unitarity and unitarity bounds in the inelastic region is known to exist [1]. But among the solutions of these papers there are no amplitudes with a Regge pole asymptotics in the physical region. More generally the methods of [1] do not allow to derive amplitudes which by construction show at least one of the following properties

i) for fixed $t$ the asymptotics in $s$ is exactly powerlike, e.g. $\operatorname{Im} A(s, 0) \simeq$ const $\cdot s$ for $|s| \rightarrow \infty$,

ii) the asymptotics in $s$ depends on the value of $t$ in the region $t<16$.

These restrictions are caused by the technique to work with the Mandelstam integral [2]. To obtain amplitudes which are dominated by Regge poles and satisfy crossing symmetry and elastic unitarity one has to evaluate elastic unitarity with an other method.

There are essentially two ways to proceed, either to use the Watson Sommerfeld representation with complex angular momenta, or to work with the Mellin transformation, i.e. the Khuri representation of the scattering amplitude [3]. In the frame work of complex angular momenta elastic unitarity becomes a trivial equation, the difficulties arise from analyticity and crossing symmetry. In a series of publications Atkinson, Warnock and their collaborators are investigating this method $[4,5]$. 
In [6] we have studied the Mellin transformation of the amplitude and explicitely calculated the transformed unitarity integral. In the present paper we shall continue this work to a complete proof of the existence of scattering amplitudes with one leading Regge pole which satisfy exactly elastic unitarity and total symmetry in $s, t$, and $u$.

The paper is organized as follows. The general definitions are presented in Section II. In Section III we introduce the Banach spaces we work with and give the norm estimates for the unitarity integral. The analytic structure of the unitarity integral in the Khuri representation with poles is studied in Section IV. If the Khuri poles correspond to exactly one Regge pole we can separate the background and the pole contribution for arbitrary Regge trajectories provided $\operatorname{Re} \alpha(s+i 0) \geqq 0$ for $4 \leqq s \leqq 16$. The condition that the Regge pole is reproduced by the unitarity interation imposes a restriction on the trajectory and the residue function which depends on the holomorphic background. In Section V we derive a non linear fixed point equation for the trajectory and the residue to implement exact elastic unitarity also for the pole contribution. This property is not guaranteed by the constructions of [4]. The $N / D$ method of [5] does not require such subsidiary constraints but there the existence of poles in the right half plane is still an open problem.

In Section VI the complete mapping for the trajectory function and the holomorphic background is given for a crossing symmetric amplitude. It is proved that the iteration of this mapping converges to a non trivial fixed point which yields the crossing symmetric and unitary amplitude with one Regge pole in the right half plane.

Technical details of our constructions have been presented in the reports $[6,7]$.

\section{The Structure of the Amplitude}

We consider the elastic scattering process of equal (pseudo) scalar particles of unit mass. The representation

$$
A(s, t)=F(s, t)+F(t, s)+F(s, u)+F(u, s)+F(u, t)+F(t, u)
$$

guarantees Mandelstam analyticity and crossing symmetry if $F(s, t)$ is a polynomially bounded function holomorphic in the variables $s$ and $t$ with only real cuts at $s \geqq 4$ and $t \geqq 16$. If the amplitude is bounded by

$$
\mid A(s, t) \leqq \operatorname{const}(1+|s|+|t|)^{\gamma_{1}}
$$

$\gamma_{1}$ real the Mellin transformation

$$
a(s, v)=\mathscr{M}\left[A_{t}(s, t)\right] \equiv \frac{1}{\pi} \int_{0}^{\infty} A_{t}(s, t) t^{-v-1} d t
$$

of the absorptive part in the $t$-channel is a holomorphic function in $v$ for $\operatorname{Re} v>\gamma_{1}$ and it is analytic in $s \in \mathbb{C}$ up to the cuts $4 \leqq s<\infty$ and $s<0$. The inverse relation

$$
A_{t}(s, t)=\mathscr{M}_{\gamma}^{-1}[a(s, v)] \equiv \frac{1}{2 i} \int_{\gamma} a(s, v) t^{v} d v
$$


is valid for any $\gamma>\gamma_{1}$ where $\int d v \ldots$ denotes the complex integration along the line $\operatorname{Re} v=\%$

In the present paper we shall assume that $a(s, v)$ can be continued to a meromorphic function in $\operatorname{Re} v>\gamma_{0},-\frac{1}{2}<\gamma_{0}<0$, with a finite number of Khuri poles [3], i.e. $F(s, t)$ can be decomposed into a polynomially increasing part $R(s, t)$ (explicitely given in Section IV) and a background $G(s, t)$ which satisfies an unsubtracted Mandelstam representation

$$
F(s, t)=R(s, t)+G(s, t) .
$$

The poles of $(2.3) a(s, v)$ are caused by $R(s, t)$ whereas $G(s, t)$ and all crossed terms in (2.1) contribute to a holomorphic background.

Under these assumptions the partial waves $f(s, l), l=0,2,4, \ldots$,

$$
A(s, t)=\sum_{l=0,2,4, \ldots}(2 l+1) f(s, l) P_{l}\left(1+\frac{2 t}{s-4}\right)
$$

can also be continued to a meromorphic function in $\operatorname{Re} l>\gamma_{0}$ and the elastic unitarity condition

$$
f(s+i 0, l)-f(s-i 0, l)=2 i \sqrt{\frac{s-4}{4 s}} f(s+i 0, l) \cdot f(s-i 0, l)
$$

is valid for $4 \leqq s \leqq 16$ in the half plane $\operatorname{Re} l>\gamma_{0}$. In [6] we have shown that Equation (2.7) is equivalent to the following condition for the Mellin transformation (2.3)

$$
\begin{aligned}
& \frac{1}{2 i}[a(s+i 0, v)-a(s-i 0, v)]=w(s, v) \\
& \text { if } 4 \leqq s \leqq 16 \text { and } \operatorname{Re} v>\gamma_{0}
\end{aligned}
$$

where the function $w(s, v)$ is defined for $s \in \mathbb{R}$ in the region $\gamma_{1} \leqq \gamma, \gamma^{\prime} \leqq \operatorname{Re} v<1+\gamma+\gamma^{\prime}$ by the unitarity integral

$$
w(s, v)=(s-4)^{v}+\sqrt{\frac{s-4}{s}} I\left(\gamma, \gamma^{\prime} ; s, v\right)
$$

with

$$
\begin{aligned}
I\left(\gamma, \gamma^{\prime} ; s, v\right)= & -\frac{1}{4 \pi^{2}} \int_{\gamma} d \xi \int_{\gamma^{\prime}} d \eta(s-4)^{\xi+\eta-2 v} \\
& \cdot M(v, \breve{\zeta}, \eta) a(s+i 0, \xi) a(s-i 0, \eta)
\end{aligned}
$$

and the kernel function

$$
M(v, \xi, \eta)=B(1+\xi, v-\xi) B(1+\eta, v-\eta) B(1+v, 1-v+\xi+\eta) .
$$

By shifting and deforming the path of integration of the integral $I\left(\gamma, \gamma^{\prime} ; s, v\right)$ the function $w(s, v)$ is calculated as meromorphic function in the half plane $\operatorname{Re} v>\gamma_{0}$ (see Sect. IV). The absorptive part (2.8) of Equation (2.3) is the Mellin transformation of the double spectral function $\varrho(s, t)$ of $A(s, t)$ which in the strip $4 \leqq s \leqq 16$ coincides with the double spectral function of $F(s, t)$. 
In the following sections we shall derive a contraction mapping for meromorphic functions $a(s, v)$ such that the fixed point of this mapping obtained by iteration satisfies the Equations (2.1)-(2.9), and the existence of poles in the half plane $\operatorname{Re} v>\gamma_{0}$ is guaranteed.

Without the difficulties caused by the poles an iteration scheme for holomorphic functions $a(s, v)$ is given by

$$
\left.\begin{array}{c}
a(s, v) \stackrel{(2.9)}{\longrightarrow} w(s, v) \stackrel{(2.4)}{\longrightarrow} \varrho^{\prime}(s, t) \underset{\text { disp.int. }}{\stackrel{\text { double }}{\longrightarrow}} F^{\prime}(s, t) \\
\stackrel{(2.1)}{\longrightarrow} A^{\prime}(s, t) \stackrel{(2.3)}{\longrightarrow} a^{\prime}(s, v) .
\end{array}\right\}
$$

These lines serve as a guidance for our construction. But due to the poles the mapping will become much more complicated and we shall present the final interation not till Section VI.

\section{Norms and Estimates}

We define a family of Banach spaces $\mathscr{E}(\lambda), \lambda \in \mathbb{R}$, of Hölder continuous functions, $s \in \mathbb{R} \rightarrow f(s) \in \mathbb{C}$, by the norm

$$
\|f(s)\|^{(\lambda)}=\sup _{\substack{s \in \mathbb{R} \\|h| \leqq 1}}(1+|s|)^{-\lambda}\left\{|f(s)|+\frac{|f(s+h)-f(s)|}{|h|^{\mu}}\right\}
$$

with a fixed Hölder index $\mu, 0<\mu<\frac{1}{2}$.

The closed subspace of $\mathscr{E}(\lambda)$ generated by functions $f(s) \in \mathscr{E}(\lambda)$ with $f(4)=0$ is denoted by $\mathscr{E}_{0}(\lambda)$.

If $f(s) \in \mathscr{E}(0)$ then

$$
f_{+}(s)=\left\{\begin{array}{lll}
f(s) & \text { if } & 4 \leqq s \leqq 16 \\
0 & \text { if } & s \notin[4,16]
\end{array}\right.
$$

is in general not an element of $\mathscr{E}(0)$. But these functions $f_{+}(s)$ generate again a Banach space denoted by $\overline{\mathscr{E}}$. Its norm $\left\|f_{+}\right\|$is given by Equation (3.1) with $\lambda=0$ and the range of $s$ and $s+h$ restricted to $4 \leqq s, s+h \leqq 16$.

The Hilbert transformation

$$
F(s)=\mathscr{H}[f] \equiv \frac{1}{\pi} \int \frac{f\left(s^{\prime}\right)}{s^{\prime}-s} d s^{\prime} \quad \text { (principal value) }
$$

maps the space $\mathscr{E}(\lambda)$ into $\mathscr{E}\left(\lambda^{\prime}\right), \lambda<0, \lambda^{\prime}>\lambda, \lambda^{\prime} \geqq-1$, with

$$
\|F(s)\|^{\left(\lambda^{\prime}\right)} \leqq \text { const } \cdot\|f(s)\|^{(\lambda)} .
$$

More details are discussed in Appendix A of [7].

The Mellin transformation and the unitarity integral can be estimated in a class of Hilbert spaces $\mathscr{L}_{\gamma}, \gamma \in \mathbb{R}$, defined by the family of norms, see [6],

$$
\|f(t)\|_{\gamma}=\left[\int_{0}^{\infty}\left|t^{-\gamma} f(t)\right|^{2}\left(1+|\log t|^{2}\right) \frac{d t}{t}\right]^{\frac{1}{2}} .
$$


The Mellin transformation

$$
a(v)=\mathscr{M}[f] \equiv \frac{1}{\pi} \int_{0}^{\infty} f(t) t^{-v-1} d t
$$

maps $\mathscr{L}_{\gamma}$ isometrically onto the Sobolev space $L_{\gamma}$ with the norm

$$
|a(v)|_{\gamma}=\left[\frac{\pi}{2} \int_{-\infty}^{+\infty} d x\left(|a(\gamma+i x)|^{2}+\left|\frac{d}{d x} a(\gamma+i x)\right|^{2}\right)\right]^{\frac{1}{2}} .
$$

A particularly interesting subspace of $\mathscr{L}_{\gamma}$ is generated by all functions $f(t) \in \mathscr{L}_{\gamma}$ with $f(t)=0$ for $t<t_{0}, 0<t_{0}<\infty$. The Mellin transformation $a(v)$ of such a function is holomorphic for $\operatorname{Re} v>\gamma$ with

$$
a(v) \in L_{\sigma} \quad \text { for } \quad \sigma \geqq \gamma
$$

and

$$
|a(v)|_{\sigma} \leqq t_{0}^{\gamma-\sigma}|a(v)|_{\gamma} .
$$

We shall denote this subspace of $L_{\gamma}$ by $L_{\gamma}^{+}\left(t_{0}\right)$ or simply by $L_{\gamma}^{+}$if $t_{0}=4$.

Spaces of functions of two variables $s$ and $t$ are introduced by a family of norms which combine the constraints (3.1) and (3.4) for $s \geqq 4$ and $t \geqq 0$

$$
\|f(s, t)\|_{\gamma, \lambda}=\sup _{\substack{s \geq 4 \\ 0 \leqq \bar{h} \leqq 1}} s^{-\lambda}\left\{\|f(s, t)\|_{\gamma}+\frac{\|f(s+h, t)-f(s, t)\|_{\gamma}}{h^{\mu}}\right\}
$$

$\lambda \in \mathbb{R}, \gamma \in \mathbb{R}$,

where $\|f(s, t)\|_{\gamma}$ is defined by Equation (3.4) with the variable $s$ as a parameter. We denote this Banach space of functions by $\mathscr{L}(\gamma, \lambda)$.

The Mellin transformation (2.2) maps $\mathscr{L}(\gamma, \lambda)$ isometrically onto the space $L(\gamma, \lambda)$ with the norm

$$
|a(s, v)|_{\gamma, \lambda}=\sup _{\substack{s \geq 4 \\ 0 \leqq h \leqq 1}} s^{-\lambda}\left\{|a(s, v)|_{\gamma}+\frac{|a(s+h, v)-a(s, v)|_{\gamma}}{h^{\mu}}\right\} .
$$

If the support of $f(s, t) \in \mathscr{L}(\gamma, \lambda)$ is restricted to $0<t_{0} \leqq t<\infty$ the Mellin transform $a(s, v)$ is holomorphic for $\operatorname{Re} v>\gamma$ and $a(s, v) \in L(\sigma, \lambda)$ for $\sigma \geqq \lambda$, the norm estimated according to Equation (3.7). This subspace of $L(\gamma, \lambda)$ is denoted by $L^{+}\left(\gamma, \lambda \mid t_{0}\right)$ or simply $L^{+}(\gamma, \lambda)$ if the value of $t_{0}=4$.

If $b(s, v) \in L(\gamma, \lambda), \lambda<0$, with $b(4, v)=0$ the Hilbert transformation

$$
a(s, v)=\mathscr{H}[b(s, v)]=\frac{1}{\pi} \int_{4}^{\infty} \frac{b\left(s^{\prime}, v\right)}{s^{\prime}-s} d s^{\prime} \quad \text { (principal value) }
$$

is a bounded linear map into $L\left(\gamma, \lambda^{\prime}\right)$ with $\lambda^{\prime}>\lambda, \lambda^{\prime} \geqq-1$.

We have not succeeded to evaluate the unitarity integral (2.9) directly. But this integral is the Mellin transformed version of the unitarity integral with the Mandelstam kernel which has been investigated in detail in the literature $[1,8]$. The results of [8] can be modified to an estimate with the norm (3.8) and we obtain (see 
[6], Sects. 3 and 5) that Equation (2.9) is a continuous mapping

$$
L\left(\gamma, \delta_{1}\right) \times L\left(\gamma, \delta_{1}\right) \rightarrow L\left(\gamma, \delta_{2}\right)
$$

with the bound

$$
|w(s, v)|_{\gamma, \delta_{2}} \leqq \text { const }|a(s+i 0, v)|_{\gamma_{1} \delta_{1}} \cdot|a(s-i 0, v)|_{\gamma, \delta_{1}}
$$

for $0<\mu<\frac{1}{2}, \gamma>-\frac{1}{2}+\mu$ and $\delta_{2}>2 \delta_{1}+\gamma$.

\section{Analytic Properties}

In the following we assume that $a(s, v)$ can be decomposed into a pole term and a background term $b(s, v)$ which is holomorphic in $v$ for $\operatorname{Re} v>\gamma_{0},-\frac{1}{2}+\mu<\gamma_{0}<0$. The Khuri poles have to be located at positions $v=\alpha(s), \alpha(s)-1, \alpha(s)-2, \ldots, \alpha(s)-M$ and the residues $\beta_{m}(s), m=0, \ldots, M$ follow from the expansion of one single Regge pole at angular momentum $l=\alpha(s)$

$$
\beta_{m}(s)=\frac{(4-s)^{m}}{m !} \frac{(-\alpha(s))_{m}(-\alpha(s))_{m}}{(-2 \alpha(s))_{m}} \beta(s) m=0,1, \ldots, M
$$

A convenient ansatz is

$$
a(s, v)=\beta(s) a_{R}(s, v)+b(s, v)
$$

with, $t_{1}=20$,

$$
\begin{aligned}
a_{R}(s, v)= & t_{1}^{\alpha(s)-v} \sum_{m=0}^{M} \frac{1}{m !}\left(\frac{s-4}{t_{1}}\right)^{m} \\
& \cdot \frac{(-\alpha(s))_{m}(-\alpha(s))_{m}}{(2 v+m+1)_{m}}\left[\frac{1}{v-\alpha+m}-\frac{1}{v+1}\right] .
\end{aligned}
$$

The trajectory function $\alpha(s)$ of the Khuri poles and the residue function $\beta(s)$ are taken as real analytic functions with Hölder continuous boundary values on the cut $4 \leqq s<\infty$. (The pole term. does not develop a left hand cut in $s$.) The background term $b(s, v)$ is holomorphic in $s$ and $v$ for $s \notin \mathbb{C}-([4, \infty) \cup(-\infty, 0])$ and $\operatorname{Re} v>\gamma_{0}$, it satisfies the reality condition $b\left(s^{*}, v^{*}\right)=b^{*}(s, v)$. The norm conditions are ${ }^{1}$

$$
\left.\begin{array}{l}
\alpha(s+i 0) \in \mathscr{E}(0), \\
\beta(s+i 0) \in \mathscr{E}(-M-1), \\
b(s+i 0, v) \in L^{+}\left(\gamma_{0}, \gamma_{1}\right)
\end{array}\right\}
$$

For the trajectory function we assume a uniform bound

$$
\|\alpha(s+i 0)\|^{(0)} \leqq \gamma_{1}-\frac{1}{2} .
$$

Then $|\alpha(s)| \leqq \gamma_{1}-\frac{1}{2}$ for $s \in \mathbb{C}_{\text {cut }}$ and the function (4.1) $a(s, v)$ is holomorphic for $v \in\left\{v \mid \operatorname{Re} v>\gamma_{0}\right.$ and $\left.|v|>\gamma_{1}\right\}$. The integer $M$ in (4.3) is chosen $\gamma_{1} \leqq M<\gamma_{1}+1$. Then $\operatorname{Re} \alpha-M<\gamma_{0}$ for $s \in \mathbb{C}_{\text {cut }}$ and all daughter trajectories which might show up in the half plane $\operatorname{Re} v>\gamma_{0}$ are contained in (4.3). The ansatz (4.3) is constructed in such a way that $a(s, v) \in L^{+}\left(\gamma_{1}, \gamma_{1}\right)$. The strong decrease of $a_{R}(s, v)$ for for $|\operatorname{Im} v| \rightarrow \infty$ like $|v|^{-2}$

1 The corresponding restrictions for the boundary values at $s-i 0$ follow from real analyticity 
guarantees that the Regge term

$$
R_{t}(s, t)=\mathscr{M}_{\gamma_{1}}^{-1}\left[\beta(s) a_{R}(s, v)\right]
$$

or

$$
\begin{aligned}
R(s, t \pm i 0)= & \sum_{m=0}^{M-1} \beta(s) a_{R}(s, m) t^{m} \\
& +\beta(s) \mathscr{M}_{\gamma}^{-1}\left[-\frac{e^{\mp i \pi v}}{\sin \pi v} a_{R}(s, v)\right]
\end{aligned}
$$

for $t \geqq 0$ with $M-1<\gamma<M, \quad \gamma \geqq \gamma_{1}-\frac{1}{2}$,

is a Hölder continuous function in both the variables $s$ and $t$. By construction of (4.3) the support of $R_{t}(s, t)$ is restricted to $t_{1}=20 \leqq t<\infty$.

Since $a(s \pm i 0, v) \in L^{+}\left(\gamma_{1}, \gamma_{1}\right)$ the unitarity integral (2.9) is defined for arbitrary $\gamma, \gamma^{\prime} \geqq \gamma_{1}$ and leads to a holomorphic function $w(s, v)$ in the space $L^{+}\left(\gamma_{1}, 3 \gamma_{1}\right)$, see Equation $(3.11)^{2}$. The analytic continuation of $w(s, v)$ into the region $\gamma_{0}<\operatorname{Re} v<\gamma_{1}$ is calculated in Appendix B of [7], here we shall present only the results. Shifting the path of integration in Equation (2.9) to the left, $\gamma_{0} \leqq \gamma, \gamma^{\prime}<\gamma_{1}$, we pass the poles of $a(s+i 0, v)$ and of $a(s-i 0, v)$. The residues contribute with terms proportional to $M(v, \alpha(s+i 0)-m, \eta)$ or/and to $M(v, \xi, \alpha(s-i 0)-m), m=0,1, \ldots, M$ hence $w(s, v)$ is a meromorphic function in $v$ for $\operatorname{Re} v>\gamma_{0}$ with poles at $v=\alpha(s+i 0)-k$ and $v=\alpha(s-i 0)-k, k=0,1,2, \ldots$.

Since the unitarity integral is diagonal in the partial wave representation the residues of the Khuri poles of $w(s, v)$ satisfy again the relation (4.1) and we can separate the pole terms and a background term in a representation similar to Equation (4.2)

$$
w(s, v)=\frac{1}{2 i}\left[\bar{\beta}(s) a_{R}(s+i 0, v)-\bar{\beta}^{*}(s) a_{R}(s-i 0, v)\right]+w_{B}(s, v) .
$$

The function $w_{B}(s, v)$ is holomorphic in $v$ for $\operatorname{Re} v>\gamma_{0}$. The residue function $\bar{\beta}(s)$ is identified at the leading pole as, see [7] Appendix B Equation (B.5),

$$
\bar{\beta}(s)=2 i \beta(s+i 0) \sqrt{\frac{s-4}{s}}(s-4)^{\alpha(s+i 0)} \varphi(s-i 0, \alpha(s+i 0))
$$

$$
\begin{aligned}
& \text { with }^{3}, \sigma=\operatorname{Re} v+\frac{1}{2}, \\
& \qquad \begin{aligned}
\varphi(s \pm i 0, v) & =B(1+v, 1+v) a(s \pm i 0, v) \\
+ & \frac{1}{2 \pi i} \int_{\sigma} d \xi(s-4)^{\xi-v} B(1+\xi, v-\xi) \\
\cdot & B(1+v, 1+\xi) a(s \pm i 0, \xi) .
\end{aligned}
\end{aligned}
$$

2 The exponential decrease of $w(s, v)$ for $\operatorname{Re} v \rightarrow \infty$ is determined by the boundary of the support of the double spectral function at $t=16+64(s-4)^{-1}>16$ and therefore the stronger results holds

$$
w(s, v) \in L^{+}\left(\gamma_{1}, 3 \gamma_{1} \mid 16\right)
$$

3 The function $2 \varphi(s, l)$ is exactly the reduced partial wave amplitude $(s-4)^{-l} f(s, l)$. We can therefore easily verify Equation (4.8) as a consequence of a Regge pole ansatz in the unitarity relation (2.7) for the partial wave amplitudes 
There are two problems with the separation (4.7). First, the analytic structure of the background $w_{B}(s, v)$ does not guarantee that it belongs to a space $L^{+}\left(\gamma_{0}, \delta\right)$. In Appendix B of [7] investigate in more detail that the leading trajectory $\alpha(s+i 0)$ is not allowed to cross the boundary at $\operatorname{Re} v=\gamma_{0}$ in order to derive a norm estimate for $w_{B}(s, v)$. Since elastic unitarity does only apply to the range $4 \leqq s \leqq 16$ we introduce a cut off function $\lambda(s) \in C^{\infty}(\mathbb{R})$

$$
\lambda(s) \begin{cases}\geqq 0 & \text { for } \quad s \in \mathbb{R} \\ =1 & \text { if } 4 \leqq s \leqq 16 \\ =0 & \text { if } \quad s \leqq 3 \text { or } s \geqq 17\end{cases}
$$

and restrict the discussion to $\lambda(s) w_{B}(s, v)$. We denote by

$$
\omega[\alpha, \beta, b](s, v)=\lambda(s) w_{B}(s, v)
$$

the map

$$
\alpha(s+i 0) \times \beta(s+i 0) \times b(s+i 0, v) \rightarrow \lambda(s) w_{B}(s, v)
$$

between the respective Banach spaces

$$
\mathscr{E}(0) \times \mathscr{E}(-M-1) \times L^{+}\left(\gamma_{0}, \gamma_{1}\right) \rightarrow L^{+}\left(\gamma_{0},-2\right)
$$

Under the conditions (4.5) and $\operatorname{Re} \alpha(s+i 0) \geqq 0$ for $4 \leqq s \leqq 17$ the following uniform norm estimates can be derived ${ }^{4}$, see Appendix B of [7],

$$
\begin{aligned}
& |\omega[\alpha, \beta, b]| \leqq \operatorname{const}(\|\beta\|+|b|)^{2}, \\
& \left|\omega\left[\alpha_{2}, \beta, b\right]-\omega\left[\alpha_{1}, \beta, b\right]\right| \\
& \quad \leqq \operatorname{const}(\|\beta\|+|b|)^{2}\left\|\alpha_{2}-\alpha_{1}\right\| \\
& \left|\omega\left[\alpha, \beta_{2}, b_{2}\right]-\omega\left[\alpha, \beta_{1}, b_{1}\right]\right| \\
& \quad \leqq \operatorname{const}\left(\left\|\beta_{1}\left|+\left\|\beta_{2}\right\|+\right| b_{1}|+| b_{2} \mid\right)\left(\left\|\beta_{2}-\beta_{1}\right\|+\left|b_{2}-b_{1}\right|\right) .\right.
\end{aligned}
$$

The second problem is more serious. The residue function $\bar{\beta}(s)$ is unambiguously defined at least in the elastic strip $4 \leqq s \leqq 16$ by Equation (4.8). But also within this limited region it is in general not the boundary value of a real analytic function. Therefore the first term in Equation (4.7) is not the discontinuity of a Khuri pole ansatz with the correct analytic structure in $s$, and an integration as proposed in Equation (2.10) would lead to cuts in the $v$-plane.

We have to modify our unitarity mapping; the necessary conditions to generate a real analytic $\bar{\beta}(s)$ are investigated in the following section.

\section{The Mapping for the Trajectory Function}

If the amplitude $A(s, t)$ satisfies elastic unitarity (2.7) it is not sufficient that the residue $\bar{\beta}(s)$ in $(4.8)$ is a real analytic function but it has to be equal to

$$
\bar{\beta}(s)=\beta(s+i 0) \text { for } 4 \leqq s \leqq 16 .
$$

$4 \quad$ Here and in the following sections we omit the labels characterizing the norms (3.1) or (3.9) if the Banach spaces are specified in the context 
This condition is equivalent to

$$
\begin{aligned}
& 2 i \sqrt{\frac{s-4}{s}}(s-4)^{\alpha} \varphi(s-i 0, \alpha)=1 \quad \text { for } \quad 4 \leqq s \leqq 16, \\
& \quad \alpha=\alpha(s+i 0)
\end{aligned}
$$

The function (4.9) $\varphi(s, v)$ is a linear transform of $a(s, v)$ and we can split it into a term $\varphi_{b}(s, v)$ generated by the background $b(s, v)$ and a term $\beta(s) \varphi_{R}(s, v)$ where $\varphi_{R}$ is calculated from the Regge ansatz (4.3) $a_{R}(s, v)$. The Equation (5.2) then reads as

$$
2 i \sqrt{\frac{s-4}{s}}(s-4)^{\alpha}\left(\beta(s-i 0) \varphi_{R}(s-i 0, \alpha)+\varphi_{b}(s-i 0, \alpha)\right)=1 .
$$

In this section we start with a given background term $b(s, v)$ and derive a mapping to determine real analytic functions $\alpha(s)$ and $\beta(s)$ with a right hand cut $s \geqq 4$ such that Equation (5.3) is fulfilled in the elastic intervall $4 \leqq s \leqq 16$.

We write $\alpha(s)$ as the sum of two terms

$$
\alpha(s)=\hat{\alpha}(s)+\alpha_{0}(s)
$$

where $\alpha_{0}(s)$ is a given real analytic function with a cut $16 \leqq s<\infty$. We assume in addition that $\alpha_{0}(s)$ is Hölder continuous on the cut, $\alpha_{0}(s+i 0) \in \mathscr{E}(0)$, and satisfies the constraints

$$
\left.\begin{array}{lll}
\alpha_{0}(4)=\sigma & \text { with } & 1<\sigma<2 \\
\operatorname{Re} \alpha_{0}(s+i 0)<-\frac{1}{2} & \text { if } & |s|>\Lambda>16 \\
\operatorname{Im} \alpha_{0}(s+i 0)>0 & \text { if } & s>16
\end{array}\right\}
$$

Only the first term $\hat{\alpha}(s)$ develops the elastic cut $s \geqq 4$. Its imaginary part is calculated from a real function $\chi(s) \in \overline{\mathscr{E}}$ as an element of $\overline{\mathscr{E}}_{0}(-2)$ by

$$
\operatorname{Im} \hat{\alpha}(s+i 0)=\sqrt{\frac{s-4}{s}}(s-4)^{\sigma} \cdot\left\{\begin{array}{llr}
0 & \text { if } & s<4 \\
\chi(s) & \text { if } & 4 \leqq s \leqq 16 \\
\chi(16) \cdot \lambda(s) & \text { if } & s>16
\end{array}\right.
$$

with the cut off $\lambda(s)$ defined in (4.10). The analytic function $\hat{\alpha}(s)$ is then given by the dispersion integral

$$
\hat{\alpha}(s)=\frac{s-4}{\pi} \int_{4}^{\infty} \frac{\operatorname{Im} \hat{\alpha}\left(s^{\prime}+i 0\right)}{\left(s^{\prime}-4\right)\left(s^{\prime}-s\right)} d s^{\prime}
$$

for $s \in \mathbb{C}-[4,17]$, the boundary values at the real axis $\hat{\alpha}(s+i 0), s \in \mathbb{R}$, are elements of $\mathscr{E}_{0}(0)$.

The Equations (5.4) and (5.7) define a continuous mapping, see [7] Appendix C

$$
\chi(s) \in \overline{\mathscr{E}} \rightarrow \alpha(s+i 0)=\alpha[\chi](s) \in \mathscr{E}(0) \text {. }
$$

If $\chi(s)$ is a small positive function the trajectory $\alpha(s)$ crosses the values $\alpha=+1$ and $\alpha=0$ at real values of $s$ below the threshold $s_{0}=4$. Due to crossing symmetry a pole of $a(s, v)$ at $\alpha(s)=1$ does not lead to a boundstate pole of the amplitude $A(s, t)$ at angular momentum $l=1$ but we have to cancel the pole at $\alpha(s)=0$ in a case like $\pi \pi$ 
scattering without boundstates. We shall therefore introduce a zero proportional $\alpha(s)$ in the residue function $\beta(s)$. Additionally the residue has to decrease like $\beta(s)$ $\sim s^{-M-1}$ for large $|s|$, more precisely (4.4) $\beta(s+i 0) \in \mathscr{E}(-M-1)$. We select a real analytic function $f(s)$ with the properties ${ }^{5}$

$$
\left.\begin{array}{l}
\operatorname{Im} f(s+i 0)=0 \quad \text { if } \quad s<18 \\
f(s+i 0) \in \mathscr{E}(-M-1) \\
f(s)>0 \text { if } \quad 3 \leqq s \leqq 17
\end{array}\right\}
$$

and define

$$
\beta(s)=\tilde{\beta}(s) \cdot f(s) \cdot \alpha(s)
$$

where $\tilde{\beta}(s)$ is a bounded real analytic function with a cut $s \geqq 4$. The imaginary part $h(s)=\operatorname{Im} \tilde{\beta}(s+i 0), s \in \mathbb{R}$, has to be a Hölder continuous function in $\mathscr{E}(0)$ with a support restricted to $4 \leqq s \leqq 18$. The real part $H(s)=\operatorname{Re} \tilde{\beta}(s+i 0)$ is related to $h(s)$ by the Hilbert transformation (3.2)

$$
H(s)=\tau+\mathscr{H}[h(s)]
$$

where $\tau$ is a (positive) real constant. To formulate Equation (5.3) in a more convenient way we introduce the functions

$$
\left.\begin{array}{c}
\mathscr{A}[\chi](s)=\left[2 i \sqrt{\frac{s-4}{s}}(s-4)^{\alpha} \varphi_{R}(s-i 0, \alpha) \alpha^{*}(s) f(s-i 0) \chi(s)\right]^{-1}, \\
\mathscr{B}[\chi, b](s)=1-2 i \sqrt{\frac{s-4}{s}}(s-4)^{\alpha} \varphi_{b}(s-i 0, \alpha),
\end{array}\right\}
$$

with

$$
\alpha=\alpha[\chi](s)=\alpha(s+i 0)
$$

where we have indicated the dependence on $\chi(s)$ and on the background $b(s, v)$. The relation (5.3) can then be written as

$$
\tilde{\beta}(s-i 0)=\mathscr{A}[\chi](s) \cdot \mathscr{B}[\chi, b](s) \cdot \chi(s) .
$$

The real and the imaginary part of $\tilde{\beta}(s+i 0)$ are now determined by

$$
\begin{aligned}
& h(s)=\left\{\begin{array}{llr}
0 & \text { if } & s \leqq 4 \\
-\left(A_{1} B_{2}+A_{2} B_{1}\right) \cdot \chi(s) & \text { if } & 4 \leqq s \leqq 16 \\
h(16) \cdot \lambda(s) & \text { if } & s \geqq 16
\end{array}\right. \\
& H(s)=\left(A_{1} B_{1}-A_{2} B_{2}\right) \cdot \chi(s) \quad \text { if } \quad 4 \leqq s \leqq 16
\end{aligned}
$$

with

$$
A_{1}=\operatorname{Re} \mathscr{A}, \quad A_{2}=\operatorname{Im} \mathscr{A}, \quad B_{1}=\operatorname{Re} \mathscr{B}, \quad B_{2}=\operatorname{Im} \mathscr{B} .
$$

Given a function $\chi(s)$ we define a real analytic residue function by the prescription

$$
\left.\begin{array}{l}
\chi \rightarrow \beta(s+i 0)=\beta[\chi, b](s): \\
\chi \stackrel{(5.13)}{\longrightarrow} h \stackrel{(5.10)}{\longrightarrow} H \stackrel{(5.9)}{\longrightarrow} \beta(s+i 0) .
\end{array}\right\}
$$

5 A possible choice is $f(s)=\int \varrho\left(s^{\prime}\right)\left(s^{\prime}-s\right)^{-M-1} d s^{\prime}$ with a real positive function $\varrho(s) \in C^{\infty}(\mathbb{R})$ the support restricted to $18 \leqq s \leqq s_{1}<\infty$ 
The notation $\beta[\chi, b]$ indicates the dependence on $\chi(s)$ and on the background term $b(s, v)$ which enters through $B_{1}$ and $B_{2}$ in Equation (5.13).

The functions $\alpha[\chi]$ and $\beta[\chi, b]$ are not yet solutions of Equation (5.3). For this purpose we define a mapping which includes also Equation (5.14)

$$
\left.\begin{array}{l}
\chi(s) \rightarrow \chi^{\prime}(s)=T[\chi, b](s): \\
\chi \stackrel{(5.13)}{\longrightarrow} h \stackrel{(5.10)}{\longrightarrow} H \stackrel{(5.14)}{\longrightarrow} \chi^{\prime}=\left(A_{1} B_{1}-A_{2} B_{2}\right)^{-1} H(s) .
\end{array}\right\}
$$

[The range of the variable $s$ in $\chi(s)$ or $\chi^{\prime}(s)$ is restricted to the intervall $4 \leqq s \leqq 16$.]

If $\chi(s)$ is a fixed point solution of the map (5.16) then $\alpha[\chi]$ and $\beta[\chi, b]$ satisfy Equations (5.9) and (5.12) and they provide a solution of Equation (5.3).

In Appendix $\mathrm{C}$ of $[7]$ the maps $\beta[\chi, b]$ and $T[\chi, b]$ have been investigated in more detail, here we give only the results. For $\chi(s) \equiv 0$ we calculate

$$
\left.\begin{array}{rl}
\beta_{0}(s+i 0) & \equiv \beta[0, b](s)=\tau \cdot f(s+i 0) \cdot \alpha_{0}(s+i 0) \\
\chi_{0}(s) & \equiv T[0,0](s) \\
& =(s-4)^{\alpha_{0}-\sigma} B\left(1+\alpha_{0}, 1+\alpha_{0}\right) \cdot \alpha_{0} \cdot f(s)
\end{array}\right\}
$$

with

$$
\alpha_{0}=\alpha_{0}(s+i 0)
$$

If $\tau>0$ the function $\chi_{0}(s)$ is positive and bounded below by $\chi_{0}(s) \geqq \tau \cdot \delta_{0}$ for $4 \leqq s \leqq 16$ with a constant $\delta_{0}$ independent of $\tau$.

For $\chi(s) \in \overline{\mathscr{E}},\|\chi\| \leqq q$, and $b(s, v) \in L^{+}\left(\gamma_{0}, \gamma_{1}\right)$ the residue function $\beta[\chi, b](s)$ comes out as an element of $\mathscr{E}(-N-1)$ with the norm estimates

$$
\left.\begin{array}{l}
\left\|\beta[\chi, b]-\beta_{0}(s)\right\| \leqq c(\tau+\|\chi\|+|b|)\|\chi\| \\
\left\|\beta\left[\chi_{2}, b\right]-\beta\left[\chi_{1}, b\right]\right\| \leqq c\left(\tau+\left\|\chi_{1}\right\|+\left\|\chi_{2}\right\|+|b|\right)\left\|\chi_{2}-\chi_{1}\right\| \\
\left\|\beta\left[\chi, b_{2}\right]-\beta\left[\chi, b_{1}\right]\right\| \leqq c\|\chi\| \cdot\left|b_{2}-b_{1}\right|
\end{array}\right\}
$$

The map $T[\chi, b]$ is a continuous transformation $\overline{\mathscr{E}} \times L^{+}\left(\gamma_{0}, \gamma_{1}\right) \rightarrow \overline{\mathscr{E}}$ only on a ball $\|\chi\| \leqq q_{1},|b| \leqq q_{2}$ because $\left(A_{1} B_{1}-A_{2} B_{2}\right)^{-1}$ can develop singularities. A detailed analysis leads to the estimates (we take $\tau>0$ )

$$
\left.\begin{array}{c}
\text { (a) }\|T[\chi, b]\| \leqq c_{1}\left(\tau+\|\chi\|^{2}+\|\chi\| \cdot|b|\right) \\
\text { (b) }\left\|T\left[\chi_{2}, b\right]-T\left[\chi_{1}, b\right]\right\| \\
\leqq c_{2}\left(\tau+\left\|\chi_{1}\right\|+\left\|\chi_{2}\right\|+|b|\right)\left\|\chi_{2}-\chi_{1}\right\| \\
\text { (c) }\left\|T\left[\chi, b_{2}\right]-T\left[\chi, b_{1}\right]\right\| \leqq c_{3}(\tau+\|\chi\|)\left|b_{2}-b_{1}\right| .
\end{array}\right\}
$$

Some consequences follow immediately from (5.17) and (5.19).

i) If we choose $0<\tau \leqq \tau_{1}=\min \left(\left(9 c_{1}^{2}\right)^{-1},\left(2 c_{2}\left(1+6 c_{1}\right)\right)^{-1}\right)$ then according to the first two estimates in $(5.19)$ the map $T[\chi, b]=\chi^{\prime}$ is a contraction on the ball $\left\{\chi \mid\|\chi\| \leqq 3 c_{1} \tau\right\}$ for any fixed $b(s, \nu)$ with

$$
|b| \leqq \sigma_{1}=\min \left(\left(3 c_{1}\right)^{-1},\left(2 c_{2}\right)^{-1}\right) \text {. }
$$


ii) If $\tau$ satisfies $\tau \leqq \delta_{0} \cdot\left(12 c_{1} c_{2}\left(1+3 c_{1}\right)\right)^{-1}=\tau_{2}$ in addition to the constraints given in i) and if $b(s, v)$ is bounded by

$$
|b| \leqq \delta_{0} \cdot\left(12 c_{1} c_{2}+4 c_{3}\right)^{-1}=\sigma_{2}
$$

then Equation (5.19) implies

$$
\|T[\chi, b]-T[0,0]\| \leqq \frac{1}{2} \tau \cdot \delta_{0}
$$

and the lower bound $\chi(s) \geqq \tau \delta_{0}$ yields

$$
T[\chi, b](s) \geqq \frac{1}{2} \tau \delta_{0} \quad \text { for } \quad 4 \leqq s \leqq 16 .
$$

Hence the convex closed set

$$
\mathscr{D}_{\tau}=\left\{\chi \in \overline{\mathscr{E}} \mid\|\chi\| \leqq 3 c_{1}, \chi(s) \geqq \frac{1}{2} \tau \cdot \delta_{0}\right\}
$$

is mapped into itself and $T$ is a contraction on $\mathscr{D}_{\tau}$ if $0<\tau \leqq \tau_{0}=\min \left(\tau_{1}, \tau_{2}\right)$ for any fixed $b(s, v)$ with $|b| \leqq \sigma_{0}=\min \left(\sigma_{.}, \sigma_{2}\right)$. The trivial fixed point solutions with $\chi(s) \equiv 0$ or $\beta(s) \equiv 0$ are excluded if $\tau>0$.

\section{The Unitarity Mapping}

We choose as independent functions $\chi(s) \in \overline{\mathscr{E}}$ and the background $b(s+i 0, v)$ $\in L^{+}\left(\gamma_{0}, \gamma_{1}\right)$ of Equation (4.2). The amplitude (2.1) can be constructed from these functions in the following way. First the Regge ansatz (4.6) is calculated with the trajectory (5.8) $\alpha[\chi](s)$ and the residue $(5.15) \beta[\chi, b](s)$. The crossed Regge terms which enter through (2.5) into (2.1) contribute with

$$
b_{R}(s, v)=\mathscr{M}\left[A b s_{t}(R(t, s)+R(t, u)+R(u, t))\right]
$$

to the background of (2.3). If $\|\chi\|$ is small enough then $\operatorname{Re} \alpha(s)<0$ for large $|s|$ and the construction (5.9) for $\beta(s)$ guarantees that $R(t, s)+R(t, u)+R(u, t) \in \mathscr{L}\left(\gamma_{0}, \gamma_{1}\right)$. In Appendix D of [7] we have estimated the dependence of these crossed Regge terms on $\alpha[\chi]$ and $\beta[\chi, b]$. The map

$$
\begin{aligned}
& \chi(s) \times b(s+i 0, v) \\
& \quad \rightarrow \Omega_{R}[\chi, b](s, v)=b_{R}(s+i 0, v) \in L^{+}\left(\gamma_{0}, \gamma_{1}\right)
\end{aligned}
$$

given by Equations (4.6), (5.8), (5.15), and (6.1) satisfies the bounds [for $\left.\chi(s) \in \mathscr{D}_{\tau}, \tau>0\right]$

$$
\left.\begin{array}{l}
\left|\Omega_{R}[\chi, b]\right| \leqq c \cdot \sqrt{\tau} \\
\left|\Omega_{R}\left[\chi_{2}, b\right]-\Omega_{R}\left[\chi_{1}, b\right]\right| \leqq c \tau^{-\frac{1}{2}}\left\|\chi_{2}-\chi_{1}\right\| \\
\left|\Omega_{R}\left[\chi, b_{2}\right]-\Omega_{R}\left[\chi, b_{1}\right]\right| \leqq c \tau^{\frac{1}{2}}\left|b_{2}-b_{1}\right| .
\end{array}\right\}
$$

These rather weak majorisations [compared to (6.7)] originate from the resonance poles which show up in $R(t, s)+R(t, u)$, see the sum in Equation (4.6).

The contribution of the background $G(s, t)$ to $(2.1)$ can be calculated from the difference $b(s, v)-b_{R}(s, v)$. The double spectral function of $G(s, t)+G(t, s)$ is the inverse Mellin transformation of $A b s_{s}\left(b(s, v)-b_{R}(s, v)\right)$. But we know from the support restrictions on the spectral functions of $R(s, t)$ and $G(s, t)$ that 
$A b s_{s} b_{R}(s, v)=0$ for $4 \leqq s \leqq 20$, hence $\mathscr{M}^{-1}\left[A b s_{s} b(s, v)\right]$ coincides for $4 \leqq s \leqq 16$ with the double spectral function of $G(s, t)$.

We are now prepared to formulate an interation prescription for the functions $\chi(s)$ and $b(s, v)$ which involves exact crossing symmetry and elastic unitarity.

The iterated function $\chi^{\prime}(s)$ is defined by the map (5.16)

$$
\chi^{\prime}(s)=T[\chi, b](s)
$$

and a new Regge ansatz (4.6) $R^{\prime}(s, t)$ is calculated with the trajectory $\alpha\left[\dot{\chi}^{\prime}\right]$ and the residue $\beta\left[\chi^{\prime}, b\right]$. Then the crossed Regge terms contribute to the background with

$$
b_{R}(s+i 0, v)=\Omega_{R}\left[\chi^{\prime}, b\right](s, v) .
$$

In Section IV we have derived the background of the unitarity integral $w_{B}(s, v)$. We calculate the dispersion integral

$$
b_{1}(s+i 0, v)=i \lambda(s) w_{B}(s, v)+\mathscr{H}\left[\lambda(s) w_{B}(s, v)\right]
$$

with the Hilbert transformation (3.10).

The transformations

$$
\left.\begin{array}{l}
\chi(s) \times b(s+i 0, v) \rightarrow \alpha[\chi], \beta[\chi, b] \stackrel{(2.9),(4.7)}{\longrightarrow} \\
\omega[\alpha, \beta, b]=\lambda(s) w_{B}(s, v) \stackrel{(6.5)}{\longrightarrow} b_{1}(s+i 0, v)
\end{array}\right\}
$$

define then a map $\chi \times b \rightarrow b_{1}(s+i 0, v)=\Omega_{1}[\chi, b](s, v)$ between the spaces $\overline{\mathscr{E}} \times L^{+}\left(\gamma_{0}, \gamma_{1}\right) \rightarrow L^{+}\left(\gamma_{0},-1\right)$ which is estimated for $\chi(s) \in \mathscr{D}_{\tau}$ as, see Equations (4.11) and (5.18),

$$
\left.\begin{array}{l}
\left|\Omega_{1}[\chi, b]\right| \leqq c(\tau+|b|)^{2} \\
\left|\Omega_{1}\left[\chi_{2}, b\right]-\Omega_{1}\left[\chi_{2}, b\right]\right| \leqq c(\tau+|b|)^{2}\left\|\chi_{2}-\chi_{1}\right\| \\
\left|\Omega_{1}\left[\gamma_{2} b_{2}\right]-\Omega_{1}\left[\chi_{2} b_{2}\right]\right| \leqq c\left(\tau+\left|b_{1}\right|+\left|b_{2}\right|\right) \cdot\left|b_{2}-b_{1}\right| .
\end{array}\right\}
$$

The function $b_{1}(s, v)$ yields the background for the iterated function $F^{\prime}(s, t)$, i.e.

$$
b_{1}(s, v)=\mathscr{M}\left[G_{t}^{\prime}(s, t)\right] \text {, }
$$

see $(2.5)$ or

$$
G^{\prime}(s, t+i 0)=\mathscr{U}_{\gamma_{0}}^{-1}\left[\frac{e^{-i \pi v}}{\sin \pi v} b_{1}(s, v)\right] \in \mathscr{L}\left(\gamma_{0} \cdot-1\right) .
$$

The crossed terms of $G^{\prime}(s, t)$ contribute with

$$
b_{2}(s, v)=\mathscr{M}\left[A b s_{t}\left(G^{\prime}(t, s)+G^{\prime}(t, u)+G^{\prime}(u, t)\right)\right]
$$

to the background of $a^{\prime}(s, v)=\mathscr{M}\left[A_{t}^{\prime}(s, t)\right]$.

The mapping $b_{1}(s, v) \rightarrow G^{\prime}(s, t) \rightarrow b_{2}(s, v)$ is a continuous linear transformation $L\left(\gamma_{0},-1\right) \rightarrow L\left(\gamma_{0}, 0\right)$. The continuity follows from simple estimates of unsubtracted dispersion integrals, see $[6,8]$. The map

$$
\begin{aligned}
& \chi(s) \times b(s+i 0, v) \\
& \quad \rightarrow \Omega[\chi, b](s, v)=b_{1}(s+i 0, v)+b_{2}(s+i 0, v) \in L\left(\gamma_{0}, 0\right)
\end{aligned}
$$


which includes the crossed terms satisfies again the estimates (6.7) (with larger constants).

The iterated background function $\left.b^{\prime}(s+i 0), v\right)$ is now defined by the sum of Equations (6.4) and (6.10) as element of $L\left(\gamma_{0}, \gamma_{1}\right)$

$$
b^{\prime}(s+i 0, v)=\Omega[\chi, b]+\Omega_{R}[T[\chi, b], b] \equiv \Phi[\chi, b] .
$$

For $\chi(s) \in \mathscr{D}_{\tau}$ and $|b| \leqq$ const this mapping is estimated by, see (5.19), (6.2), and (6.7),

(a) $|\Phi[\chi, b]| \leqq c_{4} \sqrt{\tau}$

(b) $\left|\Phi\left[\chi_{2}, b\right]-\Phi\left[\chi_{1}, b\right]\right| \leqq c_{5} \tau^{-\frac{1}{2}}(\tau+|b|)\left\|\chi_{2}-\chi_{1}\right\|$

(c) $\left|\Phi\left[\chi, b_{2}\right]-\Phi\left[\chi, b_{1}\right]\right| \leqq c_{6}\left(\sqrt{\tau}+\left|b_{1}\right|+\left|b_{2}\right|\right)\left|b_{2}-b_{1}\right|$.

The transformations (6.3) $T[\chi, b]$ and (6.11) $\Phi[\chi, b]$ define a mapping of the subset

$$
\mathscr{M}_{\tau}=\left\{\chi \times b\left|\chi \in \mathscr{D}_{\tau},\right| b \mid \leqq c_{4} \sqrt{\tau}\right\}
$$

of the Cartesian product $\overline{\mathscr{E}} \times L\left(\gamma_{0}, \gamma_{1}\right)$ into itself if $\tau$ is small enough, see Equations (5.19a) and (6.12a). This product space is again a Banach space with the norm $\|\chi(s)\|$ $+|b(s, v)|$. A fixed point of the mapping $T \times \Phi$

$$
\left.\begin{array}{l}
\tilde{\chi}=T[\tilde{\chi}, \tilde{b}] \\
\tilde{b}=\Phi[\tilde{\chi}, \tilde{b}]
\end{array}\right\}
$$

leads to an amplitude $A(s, t)$ which develops a Regge trajectory $\alpha[\tilde{\chi}](s)$ with a residue function $\beta[\tilde{\chi}, \tilde{b}](s)$ and satisfies exactly elastic unitarity and crossing symmetry. For $\tau>0$ the trivial fixed point solution with $\beta(s) \equiv 0$ is excluded, see Section V.

Unfortunately we cannot prove that $T \times \Phi$ is a contraction mapping on the convex closed set $\mathscr{M}_{\tau}$ for small values of $\tau$ because the constant on the right hand side of Equation $(6.12 b), c_{5} \tau^{-\frac{1}{2}}(\tau+|b|) \leqq c_{5} \tau^{-\frac{1}{2}}\left(\tau+c_{4} \sqrt{\tau}\right)$, is estimated by the unknown product $c_{5} \cdot c_{4}$ (whereas all other constants which determine the contraction property vanish at least like $\sqrt{\tau}$ for $\tau \rightarrow 0$ ). But the square of $T \times \Phi$

$$
\left.\left.\begin{array}{l}
\chi \\
b
\end{array}\right\} \rightarrow \begin{array}{l}
\chi^{\prime}=T[\chi, b] \\
b^{\prime}=\Phi[\chi, b]
\end{array}\right\} \rightarrow \begin{aligned}
& \chi^{\prime \prime}=T\left[\chi^{\prime}, b^{\prime}\right] \\
& b^{\prime \prime}=\Phi\left[\chi^{\prime}, b^{\prime}\right]
\end{aligned}
$$

is a contraction on $\mathscr{M}_{\tau}$ for sufficiently small $\tau$ [the difficulty in Equation (6.12b) is removed by the good behaviour of Equations (5.19) and (6.12c)]. Then the iteration

$$
\chi_{n+1} \times b_{n+1}=(T \times \Phi)\left(\chi_{n} \times b_{n}\right), \quad n=1,2, \ldots
$$

converges to the unique (non trivial) fixed point solution $\tilde{\chi} \times \tilde{b}$ within $\mathscr{M}_{\tau}$ for arbitrary input functions $\chi_{1} \times b_{1} \in \mathscr{M}_{\tau}, \tau>0$, see e.g. [9]. This completes our proof.

We would like to add some remarks about the properties of the amplitudes. The Regge trajectory $\alpha(s+i 0)=\alpha[\tilde{\chi}](s)$ of the fixed point solution has to be calculated by the iteration but we know

$$
\left|\alpha[\tilde{\chi}](s)-\alpha_{0}(s)\right| \leqq \text { const }\|\tilde{\chi}\| \leqq \text { const } 3 c_{1} \tau
$$

therefore $\alpha(s)$ will be rather close to the input function (5.5) $\alpha_{0}(s)$ if $\tau$ is small. For our construction the essential restrictions on $\alpha_{0}(s)$ are (in addition to real analyticity) 
a) boundedness

b) $\alpha_{0}(s) \geqq 0$ for $4 \leqq s \leqq 16$ [to prevent that $\alpha(s+i 0)$ crosses the boundary at $\operatorname{Re} v=\gamma_{0}$ within the elastic strip] and

c) $\operatorname{Im} \alpha_{0}(s+i 0)>0$ if $s>16$.

The boundedness is necessary only for the estimates (6.2) of the crossed channels. To incorporate indefinitely rising Regge trajectories one has to consider more complicated Regge ansätze than (4.3).

At finite energies the normalization of the trajectory is given by the value of $\alpha(4)$. The proof is done with $1<\alpha(4)<2$ but it can be extended to arbitrary positive values of $\alpha(4)$ since we do not require inelastic unitarity bounds.

The residue function $\beta(s+i 0)=\beta[\tilde{\chi}, \tilde{b}]$ (s) essentially behaves like $\beta_{0}(s+i 0)$, see Equations (5.17) and (5.18), it will therefore not vanish at values of $s$ with $\alpha(s+i 0)$ $=2,4, \ldots$ [only at $\alpha(s)=0$ we have introduced a ghost killing factor]. Hence the amplitude develops resonances with angular momenta $l=2,4, \ldots, l_{0} \leqq \max (\operatorname{Re} \alpha(s+i 0))$.

\section{References}

1. Atkinson,D.: Nucl. Phys. B7, 375 (1968); B8, 377 (1968); B13, 415 (1969); B23, 397 (1970)

Atkinson, D., Warnock, R.L.: Phys. Rev. 188, 2098 (1969)

Atkinson,D., Johnson,P.W., Warnock, R.L.: Phys. Rev. D6, 2966 (1972)

Kupsch,J.: Nucl. Phys. B12, 155 (1969); B11, 573 (1969); Nuovo Cimento 66 A, 202 (1970)

2. Mandelstam, S. : Phys. Rev. 112, 1344 (1958)

Gribov, V.N. : Proceedings of the 1960 Annual International Conference on High Energy Physics at Rochester, p. 340

3. Khuri, N.N.: Phys. Rev. 132, 914 (1963)

4. Atkinson,D., Frederiksen,J.S.: Commun. math. Phys. 40, 55 (1975)

Frederiksen, J.S. : Commun. math. Phys. 43, 1 (1975)

Frederiksen,J.S., Johnson,P.W., Warnock, R.L. : J. Math. Phys. 16, 1886 (1975)

Atkinson,D., Frederiksen,J.S., Johnson,P.W., Kaekebeke, M.: Commun. math. Phys. 51, 67 (1976)

Atkinson,D., Kaekebeke, M. : Crossing symmetric amplitudes with Regge poles. Preprint, December 1976

5. Johnson,P.W., Warnock, R. L. : Dynamical equations for a Regge theory with crossing symmetry and unitarity, Part I and II. Preprints, July 1976

6. Kupsch,J.: Unitarity integral and Mellin transformation. Preprint, November 1975

7. Kupsch,J.: On the existence of crossing symmetric and unitary scattering amplitudes with Regge poles. Technical Report, May 1977

8. Kupsch,J.: Commun. math. Phys. 19, 65 (1970)

9. Smart,D.R. : Fixed point theorems. Cambridge: University Press 1974

Communicated by J. Ginibre 
